Tumoren koordinieren. In diesem Arbeitskreis müssen retrospektiv - und besser im Ergebnis - prospektiv alle Fälle von bösartigen Tumoren des Säuglings- und Kindesalters einheitlich dokumentiert zusammengetragen, verschlüsselt und ausgewertet werden. In Heidelberg wurde zu diesem Zweck ein Erhebungsbogen ausgearbeitet und danach über 280 Fälle seit 1943 erfaßt und verschlüsselt. Der Bogen besteht aus einem doppelbögigen Blatt und umfaBt 78 Verschlüsselungspositionen; es ist noch Aufgabe des Arbeitskreises, verbindliche Klassifizierungen für das Tumorstadium zu Therapiebeginn, die Histologie und einheitliche Behandlungsfolgen zu erarbeiten; denn erst unter solchen Voraussetzungen wird es möglich sein, unsere Behandlungsergebnisse zu vergleichen, optimale Therapiekombinationen für unsere Patienten $z \mathfrak{u}$ erarbeiten und schließlich bestmögliche und reproduzierbare Heilergebnisse zu erzielen.

\title{
Zusammenfassender Bericht über die Diskussion der Vorträge 37 bis einschließlich 45
}

Zum Vortrag von Welte führt von Ekesparre-Hamburg an, daß bei nippelförmigem Anlegen einer cutanen Urethrostomie eine Stenose vermieden werden kann. Die Bricker-Blase sei im übrigen auch nicht frei von Komplikationen.

Frohmüller-Würzburg plädiert bei Verdacht auf ein embryonales Sarkom der Urogenitalorgane unbedingt für eine Cystoskopie. Anhand von drei Diapositiven wird der Fall eines 3 jährigen Jungen diskutiert, bei dem die Diagnose eines botryoiden Sarkoms cystoskopisch gestellt wurde. Es wurde mit Erfolg eine Cystektomie und Urethrosigmoideostomie vorgenommen. Welte-Köln gibt zu bedenken, daß die Cystoskopie bei Säuglingen mit großen Tumoren Schwierigkeiten bereiten und die transurethrale Gewebeentnahme histologisch zu Fehldiagnosen führen kann.

Zum Vortrag von Burghardt fügt von Ekesparre-Hamburg ergänzend hinzu, da $B$ aufgrund der Ergebnisse bei mesenchymalen Weichteiltumoren im Kindesalter weder die 2- noch die 5-Jahresgrenze eine sichere Aussage für die Überlebenschance gibt.

Zum Vortrag von Lennert über „Therapeutische Probleme bei malignen Knochentumoren" stellt Hecker-München die Frage, warum bei malignen Knochentumoren bei metastasenfreien Lymphknoten die Leistengegend bestrahlt werden sollte. Seiner Meinung nach ist die Nachbestrahlung bei freier Leistengegend nicht sinnvoll. Lennert tritt jedoch für eine Bestrahlung ein, da die Entnahme nur eines Lymphknotens keine sichere Aussage mache.

Hellwig-Köln ist zum Thema ,Sofortige Amputation oder Vorbestrahlung des Tumors" aufgrund der Erfahrungen des Tübinger Knochenregisters der Ansicht, bei Osteosarkomen oder Ewing-Sarkomen die Bestrahlung mit einer Tumorvernichtungsdosis durchzuführen. Eine primäre Amputation hält er nicht für sinnvoll, da ein großer Teil der Patienten nach primärer Amputation doch einer Lungenmetastasierung erläge.

Pieper-Heidelberg kann aufgrund des Heidelberger Materials nachweisen, daß beim Osteosarkom die Überlebenschance nach primärer Amputation mit über 25\% deutlich höher liegt als bei einer verzögerten Operation. Heeker hält die 3er-Einheit: Operation, Bestrahlung und cytostatische Behandlung auch bei Knochensarkomen für das Optimum.

Lennert spricht sich abschließend zu diesem Thema ebenfalls für eine primäre Amputation aus.

$\mathrm{Zu}$ den Vorträgen von Karrer und Pieper führt Rehbein-Bremen ergänzend an, daß die Voraussetzung für eine sinnvolle Tumordokumentation in der Gründung einer Pädiatrisch-onkologischen Gesellschaft bestehe, in der Pädiater, Kinderchirurgen, Radiologen und Pathologen zusammenarbeiten. 